[13] A. van den Bos, "The multivariate complex normal distribution-A generalization," IEEE Trans. Inf. Theory, vol. 41, no. 2, pp. 537-539, Mar. 1995.

[14] E. Ollila and V. Koivunen, "Adjusting the generalized likelihood ratio test of circularity robust to non-normality," in Proc. IEEE Int. Workshop Signal Process., Perugia, Italy, Jun. 2009.

[15] B. Picinbono, "On circularity," IEEE Trans. Signal Process., vol. 42, pp. 3473-3482, Dec. 1994.

[16] P. J. Schreier and L. L. Scharf, "Second-order analysis of improper complex random vectors and processes," IEEE Trans. Signal Process., vol. 51, pp. 714-725, Mar. 2003.

[17] J. Eriksson and V. Koivunen, "Complex random vectors and ICA models: Identifiability, uniqueness, and separability," IEEE Trans. Inf. Theory, vol. 52, no. 3, pp. 1017-1029, Mar. 2006.

[18] P. Schreier, "The degree of impropriety (noncircularity) of complex random vectors," in Proc. ICASSP, Las Vegas, NV, Apr. 2008, pp. 3909-3912.

[19] A. van den Bos, "Complex gradient and Hessian," Inst. Elect. Eng. Proc. Image Signal Process., vol. 141, pp. 380-382, Dec. 1994.

[20] T. Adalı, H. Li, M. Novey, and J.-F. Cardoso, "Complex ICA using nonlinear functions," IEEE Trans. Signal Process., vol. 56, pp. 4536-4544, Sep. 2008.

[21] M. Varanassi and B. Aazhang, "Parametric generalized Gaussian density estimation," J. Acoust. Soc. Amer., vol. 85, pp. 1404-1415, Oct. 1989.

[22] S. Haykin, Adaptive Radar Signal Processing. Hoboken, NJ: Wiley, 2007.

\section{Passive Range-Difference Estimation in a Dispersive Medium}

Y. T. Chan, Thomas K. C. Lo, and H. C. So

\begin{abstract}
Passive source localization can be achieved by utilizing the range-difference (RD) measurements between the source and several spatially separated sensors. When the signal propagation medium is nondispersive, the RD is typically obtained from time-difference-of-arrival (TDOA) between sensor outputs. However, for a dispersive medium which corresponds to in-solid localization, the TDOA information is not reliable. In this correspondence, two RD estimation algorithms for dispersive medium are derived and analyzed, assuming that the dispersion curve is available. Computer simulations are included to contrast the estimation performance of the two methods with the Cramér-Rao lower bound (CRLB) for different conditions.
\end{abstract}

Index Terms-Dispersive medium, range-difference (RD) estimation, source localization, time-difference-of-arrival (TDOA) estimation.

\section{INTRODUCTION}

A conventional approach for passive source localization is to utilize the range-difference (RD) measurements between spatially separated sensors in an array. From the RD information, a set of hyperbolic equations is constructed and the source position can then be calculated with

Manuscript received March 17, 2009; accepted September 21, 2009. First published November 06, 2009; current version published February 10, 2010. The associate editor coordinating the review of this manuscript and approving it for publication was Dr. Chong-Meng Samson See. This work was supported by a grant from CityU (Project No. 7002364).

Y. T. Chan is with the Department of Electrical and Computer Engineering, Royal Military College of Canada, Kingston, Ontario, Canada K7K 7B4.

T. K. C. Lo and H. C. So are with the Department of Electronic Engineering, City University of Hong Kong, Tat Chee Avenue, Kowloon, Hong Kong.

Color versions of one or more of the figures in this paper are available online at http://ieeexplore.ieee.org.

Digital Object Identifier 10.1109/TSP.2009.2035987 the use of the known sensor positions [1]-[4]. The minimum number of sensors required for two-dimensional positioning is three, and four for the three-dimensional case. When the signal propagation speed is a constant, the RD estimates are obtained by multiplying the velocity with the time-difference-of-arrival (TDOA) measurements, that is, the differences in arrival times between pairs of sensor outputs which receive the signal. There has been extensive research [5]-[8] on TDOA or RD estimation in nondispersive scenarios which have applications in global positioning systems [9], speaker tracking [10] and mobile phone location [11]. When the medium is dispersive, the propagation velocity is a function of frequency and the RDs cannot be obtained directly using the TDOA estimates. This is because different frequency components have different propagation speeds. Important application examples for positioning in a dispersive medium include localization of earthquake epicenters and underground explosions [12], microseismic events in mines [13], and tactile interaction in novel tangible human-computer interfaces [14], [15]. This correspondence is on the development of accurate RD estimation algorithms with known frequency-dependent velocity, denoted by $v(\omega)$ where $\omega$ is the radian frequency. Note that if $v(\omega)$ is not available, its experimental determination consists of placing sensors at a known separation distance $d$, measuring their phase-difference $\phi(\omega)$ of an input variable-frequency sinusoid, and the velocity is then computed as $v(\omega)=\omega d / \phi(\omega)$, based on different frequencies [16], [17].

There is not much literature on TDOA or RD estimation in a dispersive medium. Assuming that the TDOA is a polynomial in $\omega$, [5] finds the TDOA as the value that maximizes a weighted sum of cosine functions. The argument of the cosine is the difference between the measured phase-difference $\phi(\omega)$, and the theoretical, which is TDOA $\times \omega$. The sum is over all $\omega$ within the signal bandwidth. There is some difficulty with obtaining the optimal weights in [5], and [6] provides an improvement which is applicable when the spectral matrix satisfies an autoregressive model. As our goal is localization with a known $v(\omega)$ [16], [17], the best way is to estimate the RDs directly. The development below gives two RD estimators. The first is nonlinear which corresponds to the maximum likelihood method but requires iteration. The second is linear and thus there is a unique global solution, although its performance is slightly suboptimal.

The rest of the correspondence is organized as follows. In Section II, two RD estimation methods for a pair of sensor outputs are derived and analyzed. Both algorithms operate in the frequency domain. It is proved that their RD variances can attain the optimality benchmark, namely, the Cramér-Rao lower bound (CRLB). Extension to more than two sensors is also studied. Numerical examples are provided in Section III to evaluate the RD estimation performance in the dispersive medium. Finally, conclusions are drawn in Section IV.

\section{Algorithm DeVElopMENT}

Consider an array of $L$ sensors at known positions $\left(x_{i}, y_{i}\right), i=$ $1,2, \ldots, L$, receiving signal from a passive source at unknown position $(x, y)$ in a dispersive medium. The signal propagation velocity is a function of frequency, denoted by $v(\omega)$, and is assumed known. Let the distance between the source and the $i$ th receiver be $R_{i}=$ $\sqrt{\left(x_{i}-x\right)^{2}+\left(y_{i}-y\right)^{2}}$. The RD between the $i$ th and $j$ th receivers, denoted by $\Delta_{i j}$, is then:

$$
\begin{aligned}
\Delta_{i j} & =R_{i}-R_{j} \\
& =\sqrt{\left(x_{i}-x\right)^{2}+\left(y_{i}-y\right)^{2}}-\sqrt{\left(x_{j}-x\right)^{2}+\left(y_{j}-y\right)^{2}} .
\end{aligned}
$$

We see that the source position can be solved from the hyperbolic equations constructed from $\left\{\Delta_{i j}\right\}$. 
In the following, estimation of $\left\{\Delta_{i j}\right\}$ is addressed given signals received at the $i$ th sensor, denoted by $z_{i}(n)$, and $v(\omega)$. The $z_{i}(n)$, $n=0,1, \ldots, N-1$, are samples of its analog counterpart $z_{i}(t)$, which are expressed as

$$
z_{i}(n)=s_{i}(n)+q_{i}(n), \quad i=1,2, \ldots, L
$$

where $\left\{s_{i}(n)\right\}$ are the dispersed versions of the zero-mean Gaussian source signal, denoted by $s(n),\left\{q_{i}(n)\right\}$ are uncorrelated white Gaussian noises which are independent of $s(n)$ and $N$ is the data length received at each sensor. It is worthy to point out that the Gaussian assumption is necessary only for the CRLB derivation in Appendix A. Our algorithm development is applicable to non-Gaussian sources. Indeed, Section III contains a simulation study where the signal has a uniform probability density function (PDF). Taking the discrete Fourier transform (DFT) of $z_{i}(n)$ yields

$$
\begin{aligned}
Z_{i}(k) & =S_{i}(k)+Q_{i}(k) \\
& =S(k) e^{-j \omega_{k} \frac{R_{i}}{v\left(\omega_{k}\right)}}+Q_{i}(k), \quad k=0,1, \ldots, N-1
\end{aligned}
$$

where $S_{i}(k), S(k)$ and $Q_{i}(k)$ are the DFTs of $s_{i}(n), s(n)$, and $q_{i}(n)$, respectively, and $\omega_{k}=2 \pi k / N$. It follows from (1) and (3) that

$$
S_{i}(k)=S_{j}(k) e^{-j \omega_{k} \frac{\Delta_{i j}}{v\left(\omega_{k}\right)}}, \quad k=0,1, \ldots, N-1 .
$$

Based on (4), we first propose a frequency-domain nonlinear least squares (FNLS) estimator for $\Delta_{i j}$

$$
\begin{aligned}
\hat{\Delta}_{i j} & =\arg \min _{\tilde{\Delta}_{i j}} \sum_{k=0}^{\frac{N}{2}-1}\left|Z_{i}(k) e^{j \omega_{k} \frac{\tilde{\Delta}_{i j}}{v\left(\omega_{k}\right)}}-Z_{j}(k)\right|^{2} \\
& =\arg \max _{\widetilde{\Delta}_{i j}} \sum_{k=0}^{\frac{N}{2}-1} \operatorname{Re}\left\{Z_{i}(k) Z_{j}^{*}(k) e^{j \omega_{k} \frac{\tilde{\Delta}_{i j}}{v\left(\omega_{k}\right)}}\right\}
\end{aligned}
$$

where $\operatorname{Re}\{x\}$ denotes the real part of $x$ and ${ }^{*}$ is the conjugate operator. Let $f\left(\tilde{\Delta}_{i j}\right)=\sum_{k=0}^{N / 2-1} \operatorname{Re}\left\{Z_{i}(k) Z_{j}^{*}(k) e^{\jmath \omega_{k}\left(\tilde{\Delta}_{i j}\right) /\left(v\left(\omega_{k}\right)\right)}\right\}$. The variance of $\hat{\Delta}_{i j}$, denoted by $\operatorname{var}\left(\hat{\Delta}_{i j}\right)$, can be computed by utilizing an idea in [18] that leads to [19]

$$
\left.\operatorname{var}\left(\hat{\Delta}_{i j}\right) \approx \frac{\mathbb{E}\left\{f^{\prime 2}\left(\tilde{\Delta}_{i j}\right)\right\}}{\left(\mathbb{E}\left\{f^{\prime \prime}\left(\tilde{\Delta}_{i j}\right)\right\}\right)^{2}}\right|_{\tilde{\Delta}_{i j}=\Delta_{i j}}
$$

where $\mathbb{E}$ denotes the expectation operator, and $f^{\prime}\left(\tilde{\Delta}_{i j}\right)$ and $f^{\prime \prime}\left(\tilde{\Delta}_{i j}\right)$ are the first and second derivatives of $f\left(\tilde{\Delta}_{i j}\right)$, respectively. Let $s(n)$ be bandlimited with a constant power spectral density (PSD) between $\omega_{L} \geq 0$ and $\omega_{H} \leq \pi$ with $\omega_{H}>\omega_{L}$, and its variance be $\sigma_{s}^{2}$. We further let the powers of $q_{i}(n)$ and $q_{j}(n)$ be both equal to $\pi \sigma_{q}^{2} /\left(\omega_{H}-\omega_{L}\right)$ such that the effective noise power in $\left[\omega_{L}, \omega_{H}\right]$ is $\sigma_{q}^{2}$, then $\operatorname{var}\left(\hat{\Delta}_{i j}\right)$ is (see Appendix B)

$$
\operatorname{var}\left(\hat{\Delta}_{i j}\right) \approx \frac{\pi(1+2 \mathrm{SNR})}{N \mathrm{SNR}^{2}}\left[\int_{\omega_{L}}^{\omega_{H}}\left(\frac{\omega}{v(\omega)}\right)^{2} d \omega\right]^{-1}
$$

where $\mathrm{SNR}=\sigma_{s}^{2} / \sigma_{q}^{2}$ is the signal-to-noise ratio in $\left[\omega_{L}, \omega_{H}\right]$. From the CRLB for $\Delta_{i j}$ in Appendix A, it is seen that (7) attains the optimality benchmark for $L=2$, indicating that the FNLS method is an optimal estimator for a pair of sensor outputs.
The second approach is a linear least squares based method which follows the phase-based approach of [7]. The key idea is to estimate the phase of $S_{j}(k) / S_{i}(k)$ in (4), namely, $\omega_{k} \Delta_{i j} / v\left(\omega_{k}\right)$ for RD computation. To reduce spectral variances, we adopt the weighted overlapped segment averaging (WOSA) technique and divide the received signal $z_{i}(n)$ into $M$ segments, denoted by

$$
\begin{aligned}
& z_{i, m}(n)=z_{i}\left(n+(m-1) N_{S} O\right), \\
& \quad m=1,2, \ldots, M, \quad n=0,1, \ldots, N_{S}-1
\end{aligned}
$$

where $N_{S}$ is the length of each segment and $O$ is the overlap factor in the segmentation. In order to reduce the bias caused by duplication of the overlapped signal samples, each segment is multiplied by a smooth weighting function, denoted by $w(n)$, prior to performing DFT. As a result, the weighted DFT of $z_{i, m}(n)$, denoted by $Z_{i, m}(k)$, is

$$
\begin{array}{r}
Z_{i, m}(k)=\sum_{n=0}^{N_{S}-1} z_{i, m}(n) w(n) e^{-j \omega_{k} n}, i=1,2, \ldots, L, \\
\quad m=1,2, \ldots, M, \quad k=0,1, \ldots, N_{S}-1
\end{array}
$$

where $\omega_{k}$ is now equal to $2 \pi k / N_{S}$. Let the phase of $\sum_{m=1}^{M} Z_{i, m}^{*}(k) Z_{j, m}(k)$ be $\phi_{i j}(k)$, which is expressed as

$$
\phi_{i j}(k)=\frac{\omega_{k} \Delta_{i j}}{v\left(\omega_{k}\right)}+2 \pi b+e(k), \quad k=0,1, \ldots, N_{S}-1
$$

where the quantity $2 \pi b$, with $b$ being an integer, makes allowance for $\phi_{i j}(k)$ that can be greater than $2 \pi$. Applying a phase unwrapping algorithm to $\phi_{i j}(k)$ can determine the value of $b$. In some applications, the maximum RD, $\left|\Delta_{i j}\right|$, is known not to cause a phase wrapping in (10). Then $-\pi \leq \phi_{i j}(k) \leq \pi$ and $b=0$. For simplicity, $\phi_{i j}(k)$ will denote the unwrapped phase in the sequel. The phase errors $\{e(k)\}$ can be approximated as independent zero-mean Gaussian variables with variances [7]

$$
\sigma_{k}^{2}=\frac{\sigma_{s}^{2}}{\psi_{i j}(k)}
$$

where $\psi_{i j}(k)$ is given by

$$
\psi_{i j}(k)=\frac{\left|\gamma_{i j}(k)\right|^{2}}{1-\left|\gamma_{i j}(k)\right|^{2}}
$$

and $\gamma_{i j}(k)$ is the coherence between $Z_{i}(k)$ and $Z_{j}(k)$. An estimate for $\left|\gamma_{i j}(k)\right|^{2}$ using WOSA is

$$
\left|\gamma_{i j}(k)\right|^{2}=\frac{\left|\sum_{m=1}^{M} Z_{i, m}^{*}(k) Z_{j, m}(k)\right|^{2}}{\sum_{m=1}^{M}\left|Z_{i, m}(k)\right|^{2} \sum_{m=1}^{M}\left|Z_{j, m}(k)\right|^{2}} .
$$

Based on (10) and (11), the best linear unbiased estimator [20] for $\Delta_{i j}$ is then

$$
\hat{\Delta}_{i j}=\frac{\sum_{k=0}^{\frac{N_{S}}{2}-1} \frac{\psi_{i j}(k) \phi_{i j}(k) \omega_{k}}{v\left(\omega_{k}\right)}}{\sum_{k=0}^{\frac{N_{S}}{2}}-1} \frac{\psi_{i j}(k) \omega_{k}^{2}}{v^{2}\left(\omega_{k}\right)} .
$$

We refer to this method as the phase-based linear least squares (PLLS) estimator. Asymptotically, when $N$ and $N_{S}$ get large, the RD variance for the PLLS estimator is [5]

$$
\operatorname{var}\left(\hat{\Delta}_{i j}\right) \approx \frac{N_{S}}{2 N \sum_{k=0}^{\frac{N_{S}}{2}}-1 \frac{\psi_{i j}(k) \omega_{k}^{2}}{v^{2}\left(\omega_{k}\right)}}
$$


An approximation for (13) is given in (16), shown at the bottom of the page. When $s(n)$ has a flat spectrum, limited between $\omega_{L}$ and $\omega_{H}$, and the noise powers are identical, we have

$$
\psi_{i j}(k) \approx \begin{cases}\frac{\mathrm{SNR}^{2}}{1+2 \mathrm{SNR}}, & \omega_{k} \in\left(\omega_{L}, \omega_{H}\right) \\ 0, & \text { otherwise. }\end{cases}
$$

Substituting (17) into (15) yields

$$
\begin{aligned}
\operatorname{var}\left(\hat{\Delta}_{i j}\right) & \approx \frac{N_{S}(1+2 \mathrm{SNR})}{2 N \mathrm{SNR}^{2}}\left[\sum_{\omega_{k}=\omega_{L}}^{\omega_{H}}\left(\frac{\omega_{k}}{v\left(\omega_{k}\right)}\right)^{2}\right]^{-1} \\
& \approx \frac{\pi\left(1+2 \mathrm{SNR}^{2}\right.}{N \mathrm{SNR}^{2}}\left[\int_{\omega_{L}}^{\omega_{H}}\left(\frac{\omega}{v(\omega)}\right)^{2} d \omega\right]^{-1}
\end{aligned}
$$

which is the same as the CRLB (see Appendix A) for $L=2$, indicating its asymptotical optimality.

Section III below also considers another estimator, which is an extension of [5]. It is nonlinear and requires the maximization of

$$
\hat{\Delta}_{i j}=\arg \max _{\tilde{\Delta}_{i j}} \sum_{k=0}^{\frac{N_{S}}{2}-1} \psi_{i j}(k) \cos \left[\phi_{i j}(k)-\frac{\omega_{k} \tilde{\Delta}_{i j}}{v\left(\omega_{k}\right)}\right] .
$$

This phase-based nonlinear (PN) estimator, as well as FNLS, need multimodal searches. In our algorithm realization, Netwon-Raphson procedure is applied to find the maximum for both FNLS and PN methods, with PLLS providing the initial guess.

After obtaining the RD estimates from any of the above methods for all sensor pairs, denoted by $\hat{\boldsymbol{\Delta}}=$ $\left[\hat{\Delta}_{21}, \hat{\Delta}_{31}, \ldots, \hat{\Delta}_{L 1}, \hat{\Delta}_{32}, \ldots, \hat{\Delta}_{L(L-1)}\right]^{T}, \quad$ utilizing $\quad$ the known constraint of $\Delta_{i j}+\Delta_{j k}+\Delta_{k i}=0$ will improve the accuracy. Let $\hat{\hat{\Delta}}=\left[\hat{\hat{\Delta}}_{21}, \hat{\hat{\Delta}}_{31}, \ldots, \hat{\hat{\Delta}}_{L 1}\right]^{T}$ be the independent set, where the first sensor is selected as the reference without loss of generality. The two vectors $\hat{\Delta}$ and $\hat{\hat{\Delta}}$ are related by [21], [22]

$$
\hat{\Delta} \approx \mathbf{H} \hat{\Delta}
$$

where $\mathbf{H}=\mathbf{J K}$ with $\mathbf{K}=\left[\mathbf{0}_{(L-1) \times 1}, \mathbf{I}_{L-1}\right]^{T}$ and $\mathbf{J}=$ $\left[\mathbf{J}_{1}^{T}, \mathbf{J}_{2}^{T}, \ldots, \mathbf{J}_{L-1}^{T}\right]^{T}$ with $\mathbf{J}_{i}=\left[\mathbf{0}_{(L-i) \times(i-1)},-\mathbf{1}_{L-i}, \mathbf{I}_{L-i}\right]$, $\mathbf{I}_{i}, \mathbf{0}_{i \times j}$, and $\mathbf{1}_{i}$ represent $i \times i$ identity matrix, $i \times j$ zero matrix and column vector with length of $i$ with all its elements equal to one, respectively. The least squares solution for $\hat{\hat{\Delta}}$ is computed as

$$
\hat{\hat{\boldsymbol{\Delta}}}=\left(\mathbf{H}^{T} \mathbf{H}\right)^{-1} \mathbf{H}^{T} \hat{\boldsymbol{\Delta}} \text {. }
$$

It is interesting to note that $\hat{\hat{\Delta}}$ is an optimal estimate [22] when both the signal and noises are white Gaussian processes.

\section{Simulation RESUltS}

Computer simulations have been carried out to evaluate the accuracy of the proposed RD estimators in the presence of dispersion with known $v(\omega)$. The signal $s(n)$ is a zero-mean process of either Gaussian or uniform PDF, which is generated by passing a white

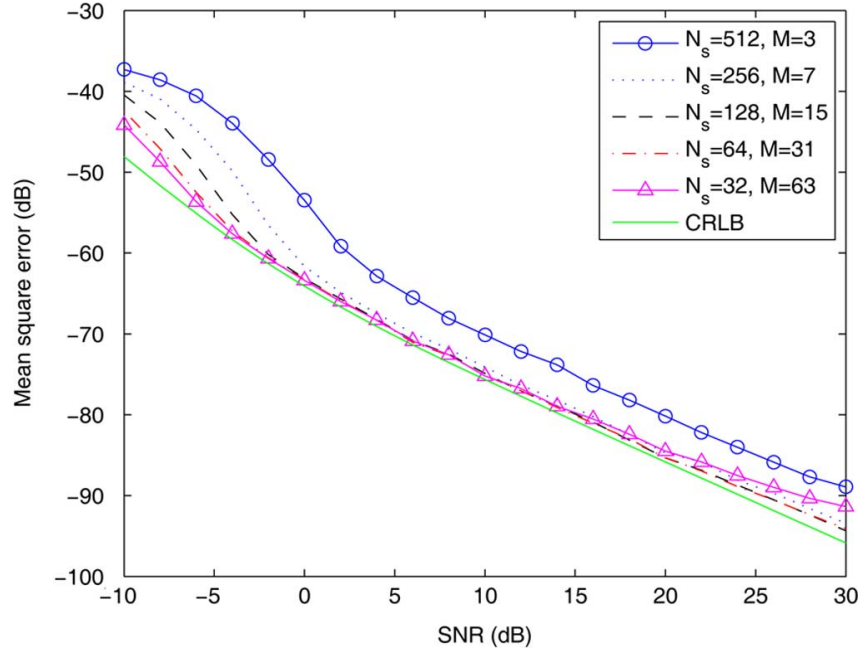

Fig. 1. MSEs of PLLS versus SNR for Gaussian signal.

sequence through an approximately rectangular-shape bandpass filter with cutoff frequencies $\omega_{L}$ and $\omega_{H}$, and has power $\sigma_{s}^{2}$. The additive noises $\left\{q_{i}(n)\right\}$ are independent and identically distributed Gaussian processes. Different SNR conditions are produced by properly scaling the noise sequences. The mean square error (MSE) in RD is used as the performance measure and comparison with the PN estimator is made. The corresponding CRLB, derived in Appendix A, is also given as an optimality benchmark. From [16], the dispersion function is approximately $v(\omega)=0.0064 \omega+130$. Performing the analog to discrete frequency conversion, the discrete velocity function is then equal to $v\left(\omega_{k}\right)=0.0064 F_{s} \omega_{k}+130$, where $F_{s}=20 \mathrm{kHz}$ is sampling frequency. In the PLLS and PN schemes, there is a 50\% overlapped segmentation, that is, $O=0.5$ in (8). The weighting function $w(n)$ is a Hanning window. Unless stated otherwise, $N=1024$. All results obtained are based on averages of 1000 independent runs.

In the first test, the effect of the segment size $N_{S}$ in the PLLS estimator is studied. A two-sensor scenario is considered and $\Delta_{21}=1.7$ $\mathrm{cm}$ is the RD. For this $\Delta_{i j}$, there is no phase wrapping in (10). Fig. 1 shows the MSEs with $N_{S}=512,256,128,64$ and 32, corresponding to $M=3,7,15,31$ and 63 , respectively, for SNR $\in[-10,30] \mathrm{dB}$. For lower SNRs, say below $10 \mathrm{~dB}$, the MSEs decrease with increasing $M$. This is because WOSA and the weighting matrix are able to suppress the noise effectively. However, for $N_{S}=32$ and SNR $>14$ $\mathrm{dB}$, and $N_{S}=64$ and $\mathrm{SNR}>27 \mathrm{~dB}$, the estimates exhibit a bias. The reason is that as the number of segments $M$ increases, for a fixed data length $N$, the segment length $N_{S}$ decreases. This in turns reduces the spectral resolution and introduces a bias. Based on the numerical results, we conclude that a good choice of $N_{S} / M$ is between 4 and 8 , giving an $\mathrm{RD}$ variance that is close to its asymptotic limit.

In the second test, we evaluate the performance of FNLS, PLLS, and PN with respect to the CRLB at different $N$. Fig. 2 plots their MSEs against SNR with $\Delta_{21}=1.7 \mathrm{~cm}$. The values of $N_{S}$ in both PLLS and

$$
\begin{aligned}
\left|\gamma_{i j}(k)\right|^{2} & \approx \frac{\left|\mathbb{E}\left\{Z_{i, m}^{*}(k) Z_{j, m}(k)\right\}\right|^{2}}{\mathbb{E}\left\{\left|Z_{i, m}(k)\right|^{2}\right\} \mathbb{E}\left\{\left|Z_{j, m}(k)\right|^{2}\right\}} \\
& \approx \frac{\left|\mathbb{E}\left\{\left|S_{m}(k)\right|^{2}\right\}\right|^{2}}{\left(\mathbb{E}\left\{\left|S_{m}(k)\right|^{2}\right\}+\mathbb{E}\left\{\left|Q_{i, m}(k)\right|^{2}\right\}\right)\left(\mathbb{E}\left\{\left|S_{m}(k)\right|^{2}\right\}+\mathbb{E}\left\{\left|Q_{j, m}(k)\right|^{2}\right\}\right)}
\end{aligned}
$$




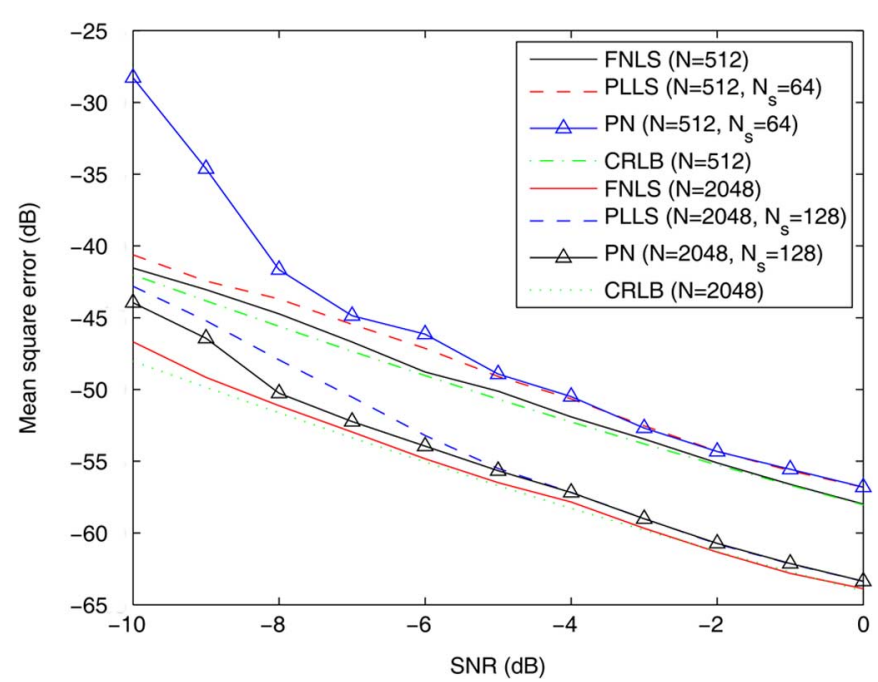

Fig. 2. MSEs of different estimators versus SNR for Gaussian signal at different observation lengths.

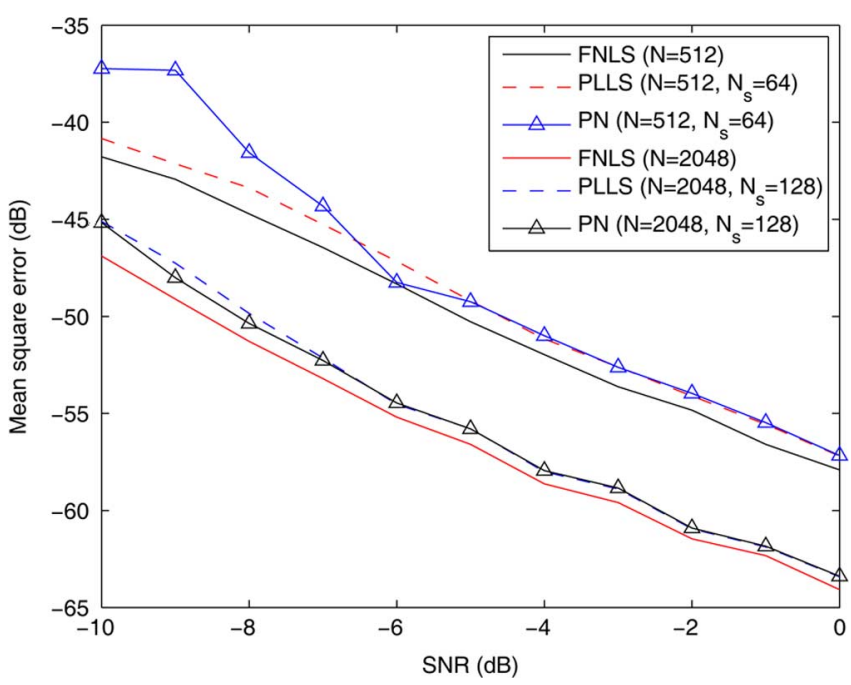

Fig. 3. Mean square errors of different estimators versus SNR for uniformly distributed signal at different observation lengths.

PN methods are $4(M+1)$ for $N=512$ and $8(M+1)$ for $N=2048$, respectively, with $M=15$. It is observed that the MSE decreases as the observation period increases in all schemes. For SNR $\in[-10,0]$ $\mathrm{dB}$, the FNLS algorithm can give performance very close to the CRLB for different values of $N$, while the other two algorithms perform very similarly and their MSEs are above the lower bound by 1 to $2 \mathrm{~dB}$. The theoretical calculation of (7) is also validated.

In the third test, we repeat the second test using a signal that has a uniform PDF. The results are shown in Fig. 3. The results are similar to that of Fig. 2, although there is no CRLB for this case.

The final test aims to study the estimation performance when the number of sensors is more than 2 . We consider $L=5$ and the true RDs are $\Delta_{21}=-1.0 \mathrm{~cm}, \Delta_{31}=-0.4 \mathrm{~cm}, \Delta_{41}=0.8 \mathrm{~cm}$, and $\Delta_{51}=1.7 \mathrm{~cm}$ where the first sensor is selected as the reference. Equation (21) is employed to give a more accurate RD estimation. We also study the performance degradation when (21) is not applied, that is, only $\left[\hat{\Delta}_{21}, \hat{\Delta}_{31}, \ldots, \hat{\Delta}_{L 1}\right]^{T}$ is used. Fig. 4 shows the average MSE performance for the four RDs and we observe that the accuracy of the

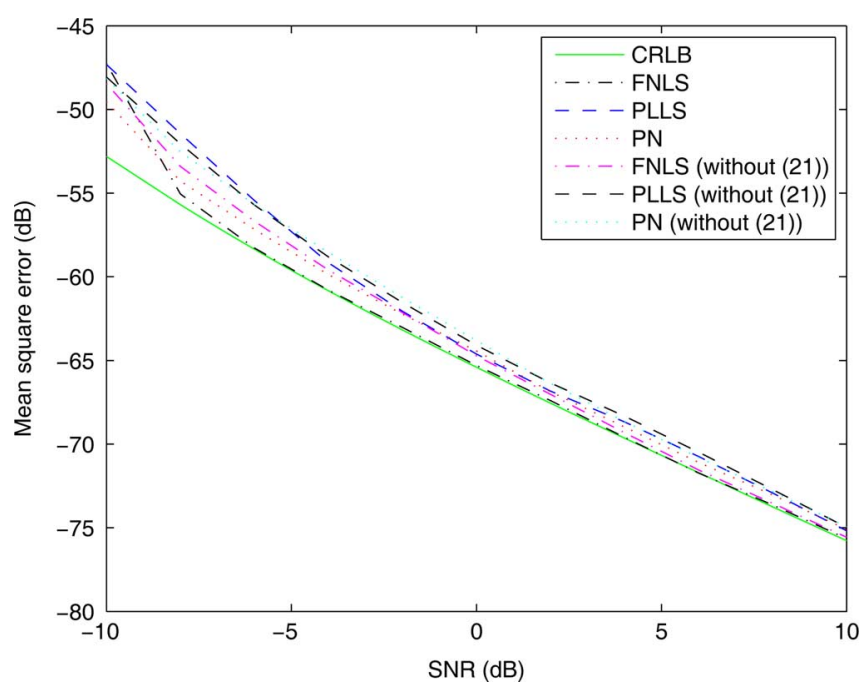

Fig. 4. Average mean square errors of different estimators versus SNR in 5 -sensor scenario.

FNLS method can meet the CRLB for the range SNR $\in[-7,0] \mathrm{dB}$. The PLLS and PN methods perform similarly and their MSEs are about 1 to $2 \mathrm{~dB}$ above the benchmark. Furthermore, it is seen that the performance of using $\left[\hat{\Delta}_{21}, \hat{\Delta}_{31}, \ldots, \hat{\Delta}_{L 1}\right]^{T}$ and $\left[\hat{\hat{\Delta}}_{21}, \hat{\hat{\Delta}}_{31}, \ldots, \hat{\hat{\Delta}}_{L 1}\right]^{T}$ is comparable for high SNR conditions, but the latter is superior when the SNR is small.

\section{CONCLUSION}

TDOA-based passive localization consists of the steps of measuring the TDOAs between sensor pairs, converting the TDOAs to RDs by multiplying by the velocity of propagation, and solving the hyperbolic equations that relate the RDs to the source position. TDOA estimation is usually achieved by cross-correlation. But if the medium is dispersive, it spreads a signal in time, and the cross-correlation function will not give a distinct peak. In addition, the signal velocity is now frequency dependent, making it difficult to perform the TDOA to RD conversion. There will be more errors when applying a nondispersive localization scheme to a dispersive medium. The severity of performance degradation will depend on the degree of dependency of velocity on frequency. As a result, it is preferably to find the RDs directly when the medium is dispersive. Assuming that the frequency-dependent velocity function is available, this correspondences gives two frequency-domain $\mathrm{RD}$ estimators. One is nonlinear and requires iterations but is optimal. The other is linear and is slightly suboptimal. The RD variances of the proposed methods are also derived in a two-sensor scenario.

\section{APPENDIX A}

The CRLB for RDs with known $v(\omega)$ is derived in the frequency domain as follows. Basically, we follow the CRLB development of [23] for passive source localization using TDOAs and gain ratios of arrival in a nondispersive medium. Starting with the continuous-time versions of $z_{i}(n), s_{i}(n)$ and $q_{i}(n)$, denoted by $z_{i}(t)$, $s_{i}(t)$, and $q_{i}(t)$, respectively, we construct a frequency domain vector $\mathbf{Z}(\omega)=\left[Z_{1}(\omega), Z_{2}(\omega), \ldots, Z_{L}(\omega)\right]^{T}$ where $Z_{i}(\omega)$ is the Fourier transform of $z_{i}(t), i=1,2, \ldots, L$. When the data record $N$ is sufficiently long, $\mathbf{Z}\left(\omega_{i}\right)$ is uncorrelated with $\mathbf{Z}\left(\omega_{j}\right)$ for $i \neq j$ [20]. Let

$$
\boldsymbol{\Delta}=\left[\Delta_{21}, \Delta_{31}, \ldots, \Delta_{L 1}\right]^{T}
$$


be the RD vector where the first sensor is assigned as the reference. The Fisher information matrix (FIM) for $\boldsymbol{\Delta}$ given $\mathbf{Z}(\omega)$, denoted by $\operatorname{FIM}(\boldsymbol{\Delta})$, is

$$
\begin{aligned}
\operatorname{FIM}(\boldsymbol{\Delta}) & =-\mathbb{E}\left\{\frac{\partial^{2} \ln p\left(\mathbf{Z}(\omega), \omega_{L} \leq \omega \leq \omega_{H}\right)}{\partial \boldsymbol{\Delta} \partial \boldsymbol{\Delta}^{T}}\right\} \\
& =-\frac{N}{2 \pi} \int_{\omega_{L}}^{\omega_{H}} \mathbb{E}\left\{\frac{\partial^{2} \ln p(\mathbf{Z}(\omega))}{\partial \boldsymbol{\Delta} \partial \boldsymbol{\Delta}^{T}}\right\} d \omega
\end{aligned}
$$

where the spectrum of $s(n)$ is nonzero for $\omega_{L} \leq \omega \leq \omega_{H}$, and $p(\mathbf{Z}(\omega))$ is the PDF of $\mathbf{Z}(\omega)$, which is of the form

$$
p(\mathbf{Z}(\omega))=\frac{1}{\pi^{L}|\mathbf{R}(\omega)|} \exp \left\{-\mathbf{Z}^{H}(\omega) \mathbf{R}^{-1}(\omega) \mathbf{Z}(\omega)\right\}
$$

where

$$
\begin{aligned}
\mathbf{R}(\omega) & =\mathbb{E}\left\{\mathbf{Z}(\omega) \mathbf{Z}^{H}(\omega)\right\} \\
& =\boldsymbol{\Phi}_{Q}(\omega)+\Phi_{S}(\omega) \mathbf{d}^{*}(\omega) \mathbf{d}^{T}(\omega)
\end{aligned}
$$

with

$$
\Phi_{Q}(\omega)=\operatorname{diag}\left[\Phi_{Q, 1}(\omega), \Phi_{Q, 2}(\omega), \ldots \Phi_{Q, L}(\omega)\right]^{T}
$$
and

$$
\mathbf{d}(\omega)=\left[1, \exp \left\{-\jmath \omega \frac{\Delta_{21}}{v(\omega)}\right\}, \ldots, \exp \left\{-\jmath \omega \frac{\Delta_{L 1}}{v(\omega)}\right\}\right]^{T}
$$

The $\Phi_{S}(\omega)$ and $\Phi_{Q, i}(\omega)$ are the power spectral densities of $s(t)$ and $q_{i}(t)$, respectively, and $|\mathbf{R}(\omega)|$ is the determinant of $\mathbf{R}(\omega)$. Note that $\boldsymbol{\Phi}_{Q}(\omega)$ is a diagonal matrix for uncorrelated $\left\{q_{i}(t)\right\}$.

For notational simplicity, the dependency on $\omega$ is removed until the final expression of $\operatorname{FIM}(\boldsymbol{\Delta})$ is attained. Taking natural logarithm on (A3) gives

$$
\ln p(\mathbf{Z})=-L \ln \pi-\ln |\mathbf{R}|-\mathbf{Z}^{H} \mathbf{R}^{-1} \mathbf{Z} .
$$

Applying Sylvester's determinant theorem [24], $|\mathbf{R}|$ is

$$
\begin{aligned}
|\mathbf{R}| & =\left(1+\Phi_{S} \mathbf{d}^{T} \boldsymbol{\Phi}_{Q}^{-1} \mathbf{d}^{*}\right)\left|\Phi_{Q}\right| \\
& =\left(1+\Phi_{S} \operatorname{tr}\left(\Phi_{Q}^{-1}\right)\right)\left|\Phi_{Q}\right|
\end{aligned}
$$

While using Woodbury's identity [25], the inverse of $\mathbf{R}$ is

$$
\mathbf{R}^{-1}=\boldsymbol{\Phi}_{Q}^{-1}-K \boldsymbol{\Phi}_{Q}^{-1} \mathbf{d}^{*} \mathbf{d}^{T} \boldsymbol{\Phi}_{Q}^{-1}
$$

where

$$
K=\frac{\Phi_{S}}{1+\Phi_{S} \operatorname{tr}\left(\Phi_{Q}^{-1}\right)} .
$$

As both $\Phi_{S}$ and $\boldsymbol{\Phi}_{Q}$ are independent of $\boldsymbol{\Delta}$, we have $(\partial \ln |\mathbf{R}|) /(\partial \boldsymbol{\Delta})=\left(\partial^{2} \ln |\mathbf{R}|\right) /\left(\partial \boldsymbol{\Delta} \partial \boldsymbol{\Delta}^{T}\right)=0$. The expression of $(\partial \ln p(\mathbf{Z})) /(\partial \boldsymbol{\Delta})$ is then simplified as

$$
\begin{aligned}
\frac{\partial \ln p(\mathbf{Z})}{\partial \boldsymbol{\Delta}} & =-\frac{\partial}{\partial \boldsymbol{\Delta}} \mathbf{Z}^{H} \mathbf{R}^{-1} \mathbf{Z} \\
& =\frac{\partial}{\partial \boldsymbol{\Delta}} K \mathbf{Z}^{H} \boldsymbol{\Phi}_{Q}^{-1} \mathbf{d}^{*} \mathbf{d}^{T} \boldsymbol{\Phi}_{Q}^{-1} \mathbf{Z} \\
& =\frac{\partial}{\partial \boldsymbol{\Delta}} K \mathbf{d}^{T} \boldsymbol{\Gamma} \mathbf{d}^{*} \\
& =K\left(\mathbf{P}+\mathbf{P}^{*}\right)
\end{aligned}
$$

where

$$
\mathbf{P}=\left(\frac{\partial \mathbf{d}}{\partial \boldsymbol{\Delta}}\right)^{T} \boldsymbol{\Gamma} \mathbf{d}^{*}
$$

and

$$
\boldsymbol{\Gamma}=\boldsymbol{\Phi}_{Q}^{-1} \mathbf{Z} \mathbf{Z}^{H} \boldsymbol{\Phi}_{Q}^{-1}
$$

Note that $\boldsymbol{\Gamma}$ is also independent of $\boldsymbol{\Delta}$. From (A6), the derivative of $\mathbf{d}$ has the form of:

$$
\frac{\partial \mathbf{d}}{\partial \boldsymbol{\Delta}}=\frac{\partial \omega}{v}\left[\begin{array}{c}
\mathbf{0}_{1 \times(L-1)} \\
\mathbf{D}_{p}
\end{array}\right]
$$

where $\mathbf{D}=\operatorname{diag}(\mathbf{d})$. For a vector and matrix, the subscript ${ }_{p}$ corresponds to the vector with its first element being removed and the matrix with its first row and column being removed, respectively. Differentiating (A11) with respect to $\boldsymbol{\Delta}^{T}$ yields

$$
\frac{\partial^{2} \ln p(\mathbf{Z})}{\partial \boldsymbol{\Delta} \partial \boldsymbol{\Delta}^{T}}=K\left(\frac{\partial \mathbf{P}}{\partial \boldsymbol{\Delta}}+\frac{\partial \mathbf{P}^{*}}{\partial \boldsymbol{\Delta}}\right)
$$

where

$$
\frac{\partial \mathbf{P}}{\partial \boldsymbol{\Delta}}=\mathbf{B}_{1}-\mathbf{B}_{2}
$$

with

and

$$
\mathbf{B}_{1}=\left(\frac{\partial \mathbf{d}}{\partial \boldsymbol{\Delta}}\right)^{T} \boldsymbol{\Gamma}\left(\frac{\partial \mathbf{d}}{\partial \boldsymbol{\Delta}}\right)^{*}
$$

$$
\mathbf{B}_{2}=\left(\frac{w}{v}\right)^{2} \operatorname{diag}\left(\left(\boldsymbol{\Gamma} \mathbf{d}^{*}\right)_{p}\right) \mathbf{D}_{p}
$$

The expected value of $\boldsymbol{\Gamma}$ is

$$
\mathbb{E}\{\boldsymbol{\Gamma}\}=\boldsymbol{\Phi}_{Q}^{-1} \mathbf{R} \boldsymbol{\Phi}_{Q}^{-1}=\boldsymbol{\Phi}_{Q}^{-1}+\Phi_{S} \boldsymbol{\Phi}_{Q}^{-1} \mathbf{d}^{*} \mathbf{d}^{T} \boldsymbol{\Phi}_{Q}^{-1} .
$$

Then we have

$$
\mathbb{E}\{\boldsymbol{\Gamma}\} \mathbf{d}^{*}=\boldsymbol{\Phi}_{N}^{-1} \mathbf{d}^{*}+\Phi_{S} \operatorname{tr}\left(\Phi_{Q}^{-1}\right) \Phi_{Q}^{-1} \mathbf{d}^{*}
$$

With the use of (A19) and (A20), $\mathbb{E}\left\{\mathbf{B}_{1}\right\}$ and $\mathbb{E}\left\{\mathbf{B}_{2}\right\}$ are computed as

$$
\begin{aligned}
\mathbb{E}\left\{\mathbf{B}_{1}\right\} & =\left(\frac{w}{v}\right)^{2} \mathbf{D}_{p}\left(\boldsymbol{\Phi}_{\mathbf{Q}_{p}}^{-1}+\Phi_{S} \mathbf{\Phi}_{\mathbf{Q}_{p}^{-1}} \mathbf{d}_{p}^{*} \mathbf{d}_{p}^{T} \boldsymbol{\Phi}_{\mathbf{Q}_{p}^{-1}}\right) \mathbf{D}_{p}^{*} \\
& =\left(\frac{w}{v}\right)^{2}\left(\boldsymbol{\Phi}_{Q_{p}^{-1}}^{-1}+\Phi_{S} \boldsymbol{\Phi}_{Q_{p}}^{-1} \mathbf{1}_{p} \mathbf{1}_{p}^{T} \boldsymbol{\Phi}_{Q_{p}}^{-1}\right) \\
\mathbb{E}\left\{\mathbf{B}_{2}\right\} & =\left(\frac{w}{v}\right)^{2}\left(\boldsymbol{\Phi}_{Q}^{-1}+\Phi_{S} \operatorname{tr}\left(\boldsymbol{\Phi}_{Q}^{-1}\right) \boldsymbol{\Phi}_{Q_{p}^{-1}}^{-1}\right) .
\end{aligned}
$$

and

Taking expectation of (A16) with the use of (A21) and (A22) yields

$$
\mathbb{E}\left\{\frac{\partial \mathbf{P}}{\partial \boldsymbol{\Delta}}\right\}=\Phi_{S}\left(\frac{\omega}{v}\right)^{2}\left(\boldsymbol{\Phi}_{Q_{p}}^{-1} \mathbf{1}_{p} \mathbf{1}_{p}^{T} \boldsymbol{\Phi}_{Q_{p}^{-1}}-\operatorname{tr}\left(\boldsymbol{\Phi}_{Q}^{-1}\right) \boldsymbol{\Phi}_{Q_{p}}^{-1}\right) .
$$

Note that $\mathbb{E}\{(\partial \mathbf{P}) /(\partial \boldsymbol{\Delta})\}^{*}=\mathbb{E}\{(\partial \mathbf{P}) /(\partial \boldsymbol{\Delta})\}$ as the vector $\boldsymbol{\Delta}$ is real. As a result, we get

$$
\begin{aligned}
& \mathbb{E}\left\{\frac{\partial^{2} \ln p(\mathbf{Z})}{\partial \boldsymbol{\Delta} \partial \boldsymbol{\Delta}^{T}}\right\}=2 K \mathbb{E}\left\{\frac{\partial \mathbf{P}}{\partial \boldsymbol{\Delta}}\right\} \\
& =2 K \Phi_{S}\left(\frac{\omega}{v}\right)^{2}\left(\boldsymbol{\Phi}_{Q_{p}^{-1}}^{-1} \mathbf{1}_{p} \mathbf{1}_{p}^{T} \boldsymbol{\Phi}_{Q_{p}^{-1}}^{-1} \operatorname{tr}\left(\boldsymbol{\Phi}_{Q}^{-1}\right) \boldsymbol{\Phi}_{Q_{p}^{-1}}^{-1}\right) .
\end{aligned}
$$


The FIM of (A2) is now expressed as

$\operatorname{FIM}(\boldsymbol{\Delta})$

$$
\begin{aligned}
= & \frac{N}{\pi} \int_{\omega_{L}}^{\omega_{H}} K(\omega) \Phi_{S}(\omega) \\
& \times\left(\operatorname{tr}\left(\boldsymbol{\Phi}_{Q}^{-1}(\omega)\right) \boldsymbol{\Phi}_{Q_{p}}^{-1}(\omega)-\boldsymbol{\Phi}_{Q_{p}}^{-1}(\omega) \mathbf{1}_{p} \mathbf{1}_{p}^{T} \boldsymbol{\Phi}_{Q_{p}}^{-1}(\omega)\right) \\
& \times\left(\frac{\omega}{v(\omega)}\right)^{2} d \omega .
\end{aligned}
$$

It is worthy to point out that when $v(\omega)=v$ which corresponds to the nondispersive scenarios, (A25) agrees with the FIM for TDOA estimation [24] up to a multiplying constant of $1 / v^{2}$. Finally, the CRLB for $\Delta_{i 1}$, denoted by $\operatorname{CRLB}\left(\Delta_{i 1}\right)$, is given by $(i-1, i-1)$ entry of the inverse of $\operatorname{FIM}(\boldsymbol{\Delta})$

$$
\operatorname{CRLB}\left(\Delta_{i 1}\right)=\left[\operatorname{FIM}^{-1}(\boldsymbol{\Delta})\right]_{i-1, i-1}, \quad i=2,3, \ldots, L .
$$

When all $\left\{q_{i}(n)\right\}$ have the same variance of $\sigma_{q}^{2}, \operatorname{FIM}(\boldsymbol{\Delta})$ can be simplified as

$\operatorname{FIM}(\boldsymbol{\Delta})=\frac{N \mathrm{SNR}^{2}}{\pi(1+L \mathrm{SNR})}\left(L \mathbf{I}_{p}-\mathbf{1}_{p} \mathbf{1}_{p}^{T}\right) \int_{\omega_{L}}^{\omega_{H}}\left(\frac{\omega}{v(\omega)}\right)^{2} d \omega$.

Using $\left(L \mathbf{I}_{p}-\mathbf{1}_{p} \mathbf{1}_{p}^{T}\right)^{-1}=\left(\mathbf{I}_{p}+\mathbf{1}_{p} \mathbf{1}_{p}^{T}\right) / L$, the corresponding CRLB is then

$$
\operatorname{CRLB}\left(\Delta_{i 1}\right)=\frac{2 \pi(1+L \mathrm{SNR})}{N L \mathrm{SNR}^{2}}\left[\int_{\omega_{L}}^{\omega_{H}}\left(\frac{\omega}{v(\omega)}\right)^{2}\right]^{-1} .
$$

In case of $v(\omega)=\alpha \omega+\beta$, we have

$$
\begin{aligned}
\int_{\omega_{L}}^{\omega_{H}} & \left(\frac{\omega}{v(\omega)}\right)^{2} d \omega \\
= & \int_{\omega_{L}}^{\omega_{H}} \frac{\omega^{2}}{(\alpha \omega+\beta)^{2}} d \omega \\
= & {\left.\left[\frac{\omega}{\alpha^{2}}-\frac{2 \beta}{\alpha^{3}} \ln (\alpha \omega+\beta)-\frac{\beta^{2}}{\alpha^{3}} \frac{1}{\alpha \omega+\beta}\right]\right|_{\omega_{L}} ^{\omega_{H}} . }
\end{aligned}
$$

\section{APPENDIX B}

In this Appendix, the variance expression of (7) for the FNLS estimator is derived. It is assumed that $s(n)$ has a constant spectrum limited between $\omega_{L}$ and $\omega_{H}$. The denominator of (6) is calculated as

$$
\begin{aligned}
& \mathbb{E}\left\{f^{\prime \prime}\left(\tilde{\Delta}_{i j}\right)\right\} \\
& =-\sum_{\omega_{k}=\omega_{L}}^{\omega_{H}} \operatorname{Re}\left\{\mathbb { E } \left\{S(k) S^{*}(k) e^{\left.\left.\jmath \omega_{k} \frac{\tilde{\Delta}_{i j}-\Delta_{i j}}{v\left(\omega_{k}\right)}\right\}\right\}\left(\frac{\omega_{k}}{v\left(\omega_{k}\right)}\right)^{2}}\right.\right. \\
& =-\frac{\pi}{\omega_{H}-\omega_{L}} N \sigma_{s}^{2} \sum_{\omega_{k}=\omega_{L}}^{\omega_{H}} \cos \left(\frac{\omega_{k}\left(\tilde{\Delta}_{i j}-\Delta_{i j}\right)}{v\left(\omega_{k}\right)}\right)\left(\frac{\omega_{k}}{v\left(\omega_{k}\right)}\right)^{2} .
\end{aligned}
$$

Substituting $\tilde{\Delta}_{i j}=\Delta_{i j}$ yields

$$
\left.\mathbb{E}\left\{f^{\prime \prime}\left(\tilde{\Delta}_{i j}\right)\right\}\right|_{\tilde{\Delta}_{i j}=\Delta_{i j}}=-\frac{\pi}{\omega_{H}-\omega_{L}} N \sigma_{s}^{2} \sum_{\omega_{k}=\omega_{L}}^{\omega_{H}}\left(\frac{\omega_{k}}{v\left(\omega_{k}\right)}\right)^{2} .
$$

The numerator of (6) is derived in (B3)-(B5).

$$
\begin{aligned}
\mathbb{E}\left\{f^{\prime 2}\left(\tilde{\Delta}_{i j}\right)\right\} & \sum_{\omega_{k}=\omega_{L}}^{\omega_{H}} \sum_{\omega_{l}=\omega_{L}}^{\omega_{H}} \mathbb{E}\left\{\operatorname { R e } \left\{\frac{\jmath \omega_{k}}{v\left(\omega_{k}\right)} Z_{i}(k) Z_{j}^{*}(k) e^{\left.\jmath \omega_{k} \frac{\tilde{\Delta}_{i j}}{v\left(\omega_{k}\right)}\right\}}\right.\right. \\
& \left.\times \operatorname{Re}\left\{\frac{\jmath \omega_{l}}{v\left(\omega_{l}\right)} Z_{i}(l) Z_{j}^{*}(l) e^{\jmath \omega_{l} \frac{\tilde{\Delta}_{i j}}{v\left(\omega_{l}\right)}}\right\}\right\} \\
= & \frac{1}{4}\left(2 C_{1}-C_{2}-C_{3}\right)
\end{aligned}
$$

where

$$
\begin{aligned}
C_{1}= & \sum_{\omega_{k}=\omega_{L}}^{\omega_{H}} \sum_{\omega_{l}=\omega_{L}}^{\omega_{H}} \mathbb{E}\left\{Z_{i}(k) Z_{i}^{*}(l) Z_{j}^{*}(k) Z_{j}(l) e^{x\left(\frac{\omega_{k}}{v\left(\omega_{k}\right)}-\frac{\omega_{l}}{v\left(\omega_{l}\right)}\right) \tilde{\Delta}_{i j}}\right\} \\
& \times \frac{\omega_{k}}{v\left(\omega_{k}\right)} \frac{\omega_{l}}{v\left(\omega_{l}\right)} \\
C_{2}= & \sum_{\omega_{k}=\omega_{L}}^{\omega_{H}} \sum_{\omega_{l}=\omega_{L}}^{\omega_{H}} \mathbb{E}\left\{Z_{i}(k) Z_{i}(l) Z_{j}^{*}(k) Z_{j}^{*}(l) e^{-\jmath\left(\frac{\omega_{k}}{v\left(\omega_{k}\right)}+\frac{\omega_{l}}{v\left(\omega_{l}\right)}\right) \tilde{\Delta}_{i j}}\right\}
\end{aligned}
$$

and

$$
\begin{aligned}
& C_{1}=\sum_{\omega_{k}=\omega_{L}}^{\omega_{H}} \sum_{\omega_{l}=\omega_{L}}^{\omega_{H}}\left[\mathbb{E}\left\{S(k) S^{*}(k)\right\} E\left\{S(l) S^{*}(l)\right\} e^{\jmath\left(\frac{\omega_{k}}{v\left(\omega_{k}\right)}-\frac{\omega_{l}}{v\left(\omega_{l}\right)}\right)\left(\tilde{\Delta}_{i j}-\Delta_{i j}\right)}\right. \\
& +\mathbb{E}\left\{S(k) S^{*}(l)\right\} E\left\{S^{*}(k) S(l)\right\} e^{j\left(\frac{\omega_{k}}{v\left(\omega_{k}\right)}-\frac{\omega_{l}}{v\left(\omega_{l}\right)}\right)\left(\tilde{\Delta}_{i j}-\Delta_{i j}\right)} \\
& +\mathbb{E}\{S(k) S(l)\} E\left\{S^{*}(k) S^{*}(l)\right\} e^{j\left(\frac{\omega_{k}}{v\left(\omega_{k}\right)}-\frac{\omega_{l}}{v\left(\omega_{l}\right)}\right)\left(\tilde{\Delta}_{i j}-\Delta_{i j}\right)} \\
& +\mathbb{E}\left\{S(k) S^{*}(l)\right\} E\left\{Q_{i}^{*}(k) Q_{i}(l)\right\} e^{j\left(\frac{\omega_{k}}{v\left(\omega_{k}\right)}-\frac{\omega_{l}}{v\left(\omega_{l}\right)}\right) \tilde{\Delta}_{i j}} \\
& +\mathbb{E}\left\{S^{*}(k) S(l)\right\} E\left\{Q_{j}(k) Q_{j}^{*}(l)\right\} e^{j\left(\frac{\omega_{k}}{v\left(\omega_{k}\right)}-\frac{\omega_{l}}{v\left(\omega_{l}\right)}\right)\left(\tilde{\Delta}_{i j}-\Delta_{i j}\right)} \\
& \left.+\mathbb{E}\left\{S(k) S^{*}(l)\right\} E\left\{Q_{j}(k) Q_{j}^{*}(l)\right\} e^{j\left(\frac{\omega_{k}}{v\left(\omega_{k}\right)}-\frac{\omega_{l}}{v\left(\omega_{l}\right)}\right) \tilde{\Delta}_{i j}}\right] \frac{\omega_{k}}{v\left(\omega_{k}\right)} \frac{\omega_{l}}{v\left(\omega_{l}\right)} \\
& \mathbb{E}\left\{\left(S(k) S^{*}(l)\right\}=\mathbb{E}\left\{\sum_{n=0}^{N-1} s(n) e^{-\jmath \frac{2 \pi n k}{N}} \sum_{m=0}^{N-1} s(m) e^{\jmath \frac{2 \pi m l}{N}}\right\}\right. \\
& = \begin{cases}\frac{\pi}{\omega_{H}-\omega_{L}} N \sigma_{s}^{2}, & l=k \text { and } \omega_{k}, \omega_{l} \in\left(\omega_{L}, \omega_{H}\right) \\
0, & \text { otherwise }\end{cases}
\end{aligned}
$$

$$
\begin{aligned}
\mathbb{E}\{(S(k) S(l)\}= & \mathbb{E}\left\{\sum_{n=0}^{N-1} s(n) e^{-\jmath \frac{2 \pi n k}{N}} \sum_{m=0}^{N-1} s(m) e^{-\jmath \frac{2 \pi m l}{N}}\right\} \\
& = \begin{cases}\frac{\pi}{\omega_{H}-\omega_{L}} N \sigma_{s}^{2}, & l=k=0 \text { and } \omega_{k}, \omega_{l} \in\left(\omega_{L}, \omega_{H}\right) \\
0, & \text { otherwise. }\end{cases}
\end{aligned}
$$




$$
\begin{aligned}
& \times \frac{\omega_{k}}{v\left(\omega_{k}\right)} \frac{\omega_{l}}{v\left(\omega_{l}\right)} \\
C_{3}= & \sum_{\omega_{k}=\omega_{L}}^{\omega_{H}} \sum_{\omega_{l}=\omega_{L}}^{\omega_{H}} \mathbb{E}\left\{Z_{i}^{*}(k) Z_{i}^{*}(l) Z_{j}(k) Z_{j}(l) e^{\jmath\left(\frac{\omega_{k}}{v\left(\omega_{k}\right)}+\frac{\omega_{l}}{v\left(\omega_{l}\right)}\right) \tilde{\Delta}_{i j}}\right\} \\
& \times \frac{\omega_{k}}{v\left(\omega_{k}\right)} \frac{\omega_{l}}{v\left(\omega_{l}\right)} .
\end{aligned}
$$

The term $C_{1}$ is expanded as (B5)-(B7), shown at the bottom of the previous page. Similarly, we have

$$
\begin{aligned}
& \mathbb{E}\left\{\left(Q_{i}(k) Q_{i}^{*}(l)\right\}\right. \\
& \quad=\left\{\begin{array}{ll}
\frac{\pi}{\omega_{H}-\omega_{L}} N \sigma_{q}^{2}, & l=k \\
0, & \text { otherwise }
\end{array}, \quad i=1,2, \ldots, L\right.
\end{aligned}
$$

and

$$
\begin{aligned}
& \mathbb{E}\left\{\left(Q_{i}(k) Q_{i}(l)\right\}\right. \\
& \quad=\left\{\begin{array}{ll}
\frac{\pi}{\omega_{H}-\omega_{L}} N \sigma_{q}^{2}, & l=k=0 \\
0, & \text { otherwise. }
\end{array}, \quad i=1,2, \ldots, L\right.
\end{aligned}
$$

Substituting $\tilde{\Delta}_{i j}=\Delta_{i j}$ and using (B6) to (B9), $C_{1}$ becomes

$$
\begin{array}{r}
C_{1}=\frac{\pi^{2}}{\left(\omega_{H}-\omega_{L}\right)^{2}} N^{2}\left(\sigma_{s}^{4} \sum_{\omega_{k}=\omega_{L}}^{\omega_{H}} \sum_{\omega_{l}=\omega_{L}}^{\omega_{H}} \frac{\omega_{k}}{v\left(\omega_{k}\right)} \frac{\omega_{l}}{v\left(\omega_{l}\right)}\right. \\
\left.+\left(\sigma_{s}^{4}+2 \sigma_{s}^{2} \sigma_{q}^{2}+\sigma_{q}^{4}\right) \sum_{\omega_{k}=\omega_{L}}^{\omega_{H}}\left(\frac{\omega_{k}}{v\left(\omega_{k}\right)}\right)^{2}\right) .
\end{array}
$$

In a similar manner, when $\tilde{\Delta}_{i j}=\Delta_{i j}, C_{2}$ and $C_{3}$ are calculated as

$$
\begin{array}{r}
C_{2}=C_{3}=\frac{\pi^{2}}{\left(\omega_{H}-\omega_{L}\right)^{2}} N^{2}\left(\sigma_{s}^{4} \sum_{\omega_{k}=\omega_{L}}^{\omega_{H}} \sum_{\omega_{l}=\omega_{L}}^{\omega_{H}} \frac{\omega_{k}}{v\left(\omega_{k}\right)} \frac{\omega_{l}}{v\left(\omega_{l}\right)}\right. \\
\left.+\sigma_{s}^{4} \sum_{\omega_{k}=\omega_{L}}^{\omega_{H}}\left(\frac{\omega_{k}}{v\left(\omega_{k}\right)}\right)^{2}\right) .
\end{array}
$$

Substituting (B10) and (B11) into (B3) yields

$$
\begin{aligned}
\mathbb{E} & \left.\left\{f^{\prime 2}\left(\tilde{\Delta}_{i j}\right)\right\}\right|_{\tilde{\Delta}_{i j}=\Delta_{i j}} \\
& =\frac{1}{2} \frac{\pi^{2}}{\left(\omega_{H}-\omega_{L}\right)^{2}} N^{2}\left(2 \sigma_{s}^{2} \sigma_{q}^{2}+\sigma_{q}^{4}\right) \sum_{\omega_{k}=\omega_{L}}^{\omega_{H}}\left(\frac{\omega_{k}}{v\left(\omega_{k}\right)}\right)^{2}
\end{aligned}
$$

With the use of (B2) and (B12), (6) becomes

$$
\begin{aligned}
\operatorname{var}\left(\hat{\Delta}_{i j}\right) & \approx \frac{1+2 \mathrm{SNR}}{2 \mathrm{SNR}^{2}}\left[\sum_{\omega_{k}=\omega_{L}}^{\omega_{H}}\left(\frac{\omega_{k}}{v\left(\omega_{k}\right)}\right)^{2}\right]^{-1} \\
& \approx \frac{\pi\left(1+2 \mathrm{SNR}^{2}\right.}{N \mathrm{SNR}^{2}}\left[\int_{\omega_{L}}^{\omega_{H}}\left(\frac{\omega}{v(\omega)}\right)^{2} d \omega\right]^{-1}
\end{aligned}
$$

\section{REFERENCES}

[1] J. O. Smith and J. S. Abel, "Closed-form least-squares source location estimation from range-difference measurements," IEEE Trans. Acoust., Speech, Signal Process., vol. 35, pp. 1661-1669, Dec. 1987.

[2] B. Friedlander, "A passive localization algorithm and its accuracy analysis," IEEE J. Ocean. Eng., vol. 12, pp. 234-245, Jan. 1987.

[3] Y. T. Chan and K. C. Ho, "A simple and efficient estimator for hyperbolic location," IEEE Trans. Signal Process., vol. 42, no. 8, pp. 1905-1915, Aug. 1994.

[4] Y. Huang, J. Benesty, G. W. Elko, and R. Mersereau, "Real-time passive source localization: A practical linear-correction least-squares approach," IEEE Trans. Speech Audio Process., vol. 9, no. 8, pp. 943-956, Nov. 2001.

[5] B. V. Hamon and E. J. Hannan, "Spectral estimation of time delay for dispersive and non-dispersive systems," Appl. Statist., vol. 23, no. 2, pp. 134-142, 1974

[6] E. J. Hannan and P. J. Thomson, "Delay estimation and the estimation of coherence and phase," IEEE Trans. Acoust., Speech, Signal Process., vol. ASSP-29, no. 3, Jun. 1981.

[7] Y. T. Chan, R. V. Hattin, and J. B. Plant, "The least squares estimation of time delay and its use in signal detection," IEEE Trans. Acoust., Speech, Signal Process., vol. 29, pp. 577-581, Jun. 1981.

[8] G. C. Carter, Coherence and Time Delay Estimation: An Applied Tutorial for Research, Development, Test, and Evaluation Engineers. Piscatway, NJ: IEEE Press, 1993.

[9] J. B. Tsui, Fundamentals of Global Positioning System Receivers. New York: Wiley, 2000.

[10] Acoustic Signal Processing for Telecommunication, S. L. Gay and J. Benesty, Eds. Boston, MA: Kluwer Academic, 2000.

[11] J. J. Caffery, Jr., Wireless Location in CDMA Cellular Radio Systems. Boston, MA: Kluwer Academic, 2000.

[12] F. Klein, "Finding an earthquakes location with modern seismic networks," Earthquake Hazards Program Northern California Oct. 2000.

[13] B. L. F. Daku, J. E. Salt, L. Sha, and A. F. Prugger, "An algorithm for locating microseismic events," in Proc. IEEE Canad. Conf. Electrical Comput. Eng., Niagara Falls, Canada, May 2004, vol. 4, pp. 2311-2314.

[14] G. De Sanctis, D. Rovetta, A. Sarti, G. Scarparo, and S. Tubaro, "Localization of tactile interactions through TDOA analysis: Geometric vs. inversion-based method," presented at the 2006 Eur. Signal Process. Conf., Florence, Italy, Sep. 2006.

[15] TAI-CHI: Tangible Acoustic Interfaces for Computer-Human Interaction, [Online]. Available: http://www.taichi.cf.ac.uk

[16] D. Rovetta, A. Sarti, G. De Sanctis, and M. Fabiani, "Modelling elastic wave propagation in thin plates," presented at the 2006 Eur. Signal Process. Conf., Florence, Italy, Sep. 2006.

[17] C. B. Park, R. D. Miller, and J. Xia, "Multichannel analysis of surface waves," Geophysics, vol. 64, no. 3, pp. 800-808, May-June 1999.

[18] V. H. MacDonald and P. M. Schultheiss, "Optimum passive bearing estimation in a spatially incoherent noise environment," J. Acoust. Soc. Amer., vol. 46, no. 1, pp. 37-43, 1969.

[19] J. P. Ianniello, "Large and small error performance limits for multipath time delay estimation," IEEE Trans. Acoust., Speech, Signal Process., vol. 34, no. 2, pp. 245-251, Apr. 1986

[20] S. M. Kay, Fundamentals of Statistical Signal Processing: Estimation Theory. Englewood Cliffs, NJ: Prentice-Hall, 1993.

[21] R. O. Schmidt, "Least squares range difference location," IEEE Trans. Aerosp. Electron. Syst., vol. 32, no. 1, pp. 234-242, Jan. 1996.

[22] H. C. So, Y. T. Chan, and F. K. W. Chan, "Closed-form formulae for optimum time difference of arrival based localization," IEEE Trans. Signal Process., vol. 56, no. 6, pp. 2614-2620, Jun. 2008.

[23] K. C. Ho and M. Sun, "Passive source localization using time differences of arrival and gain ratios of arrival," IEEE Trans. Signal Process. vol. 56, no. 2, pp. 464-477, Feb. 2008

[24] W. Hahn and S. Tretter, "Optimum processing for delay-vector estimation in passive signal arrays," IEEE Trans. Inf. Theory, vol. IT-19, no. 5, pp. 608-614, Sep. 1973.

[25] L. L. Scharf, Statistical Signal Processing: Detection, Estimation and Time Series Analysis. Reading, MA: Addison-Wesley, 1991.

which is (7). 